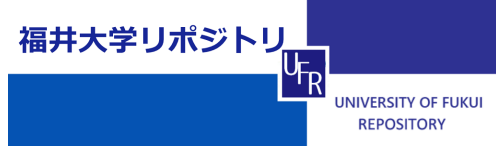

Chemi cal Ti mescal e of Dar $\mathrm{k} \mathrm{Cl}$ oud Cor es Est i mat ed from Deut er i um Fr act i onat i on

\begin{tabular}{|l|l|}
\hline 著者 & $\begin{array}{l}\text { SAl TO Shuj i, Al KAWA Yur i, HERBST Er i c, OH SH } \\
\text { Nasat oshi , H ROTA Tonmya, YAMAMOTO Sat oshi , } \\
\text { KAl FU Nor i o }\end{array}$ \\
\hline $\begin{array}{l}\text { j our nal or } \\
\text { publ i cat i on t i t e }\end{array}$ & Ast r ophysi cal J our nal \\
\hline vol une & 569 \\
\hline page range & $836-840$ \\
\hline year & $2002-04$ \\
\hline URL & ht t p: //hdl . handl e. net /10098/1586 \\
\hline
\end{tabular}




\title{
CHEMICAL TIMESCALE OF DARK CLOUD CORES ESTIMATED FROM DEUTERIUM FRACTIONATION
}

\author{
Shuji Saito, ${ }^{1}$ Yuri Aikawa, ${ }^{2}$ Eric Herbst, ${ }^{3}$ Masatoshi Ohishi, ${ }^{4}$ Tomoya Hirota,
} SATOSHi Yamamoto, ${ }^{6}$ AND NORIO KAlFU ${ }^{4}$

\begin{abstract}
The chemical evolutionary age of dark cloud cores is estimated from the degree of deuterium fractionation, on the assumption that the chemical model used is reasonable. The method is applied to dark cloud cores along the $\mathrm{TMC}-1$ ridge, using the abundance ratios between $\mathrm{DCO}^{+}$and $\mathrm{H}^{13} \mathrm{CO}^{+}$combined with the new standard model network of gas-phase chemical reactions. The difference in deuterium fractionation between the ammonia peak and the cyanopolyyne peak, though its error transferred from the observed data is relatively large, is explained by a time difference in the evolutionary age of more than $10^{5} \mathrm{yr}$, or by a small change in the depletion factor of carbon and oxygen, which also indicates the degree of core evolution. The method of determining the evolutionary age of dark cloud cores is somewhat free from the details of chemical reaction not directly related to deuterium fractionation. The present result is compared with those from other methods.
\end{abstract}

Subject headings: astrochemistry — ISM: abundances — ISM: clouds - ISM: molecules

\section{INTRODUCTION}

More than 120 molecular species have been detected and identified in space. The mechanism of their production and destruction has been proposed, and chemical models have been developed to explain and predict the existence and abundance of various interstellar molecules (for example, Herbst \& Leung 1989, 1990; Lee, Bettens, \& Herbst 1996). The predicted abundances of some main species are in agreement with observed values, but for many other molecules, especially heavy atom-bearing molecules and large polyatomic molecules, there is occasionally a large difference between the predicted and observed abundances. Revisions of chemical models have been made by including the grain-surface chemistry (Hasegawa \& Herbst 1993), neutral-neutral reactions (Herbst et al. 1994), and grain-surface molecular depletion and desorption (Bergin, Langer, \& Goldsmith 1995). Using a time-dependent chemical model, Suzuki et al. (1992) proposed that the relative magnitude of molecular column densities can be interpreted as an effect of chemical evolution of dark clouds, and the ratio [CCS]/ $\left[\mathrm{NH}_{3}\right]$ may serve as an indicator of cloud evolution.

Hirahara et al. (1992) carried out high-resolution mapping observations of carbon-chain molecules and $\mathrm{NH}_{3}$ along the ridge of TMC-1 and proposed that the difference in molecular distribution of CCS and $\mathrm{NH}_{3}$ between the cyanopolyyne peak and the ammonia peak can be ascribed to their different stages of chemical evolution. On the basis of a one-dimensional hydrodynamical simulation of a self-gravi-

${ }^{1}$ Research Center for Development of Far-Infrared Region, Fukui University, 3-9-1 Bunkyo, Fukui 910-8507, Japan.

${ }^{2}$ Department of Earth and Planetary Sciences, Faculty of Science, Kobe University, Kobe 657-8501, Japan.

${ }^{3}$ Department of Physics and Department of Astronomy, The Ohio State University, Columbus, OH 43210.

${ }^{4}$ National Astronomical Observatory of Japan, Mitaka, Tokyo 181 8588 , Japan.

${ }^{5}$ Department of Physics, Kagoshima University, Kagoshima 890-0065, Japan.

6 Department of Physics, The University of Tokyo, Bunkyo-ku, Tokyo 113-0033, Japan. tating gas cloud, Hanawa, Yamamoto, \& Hirahara (1994) proposed a sequential core collapse along the ridge of TMC-1. Howe, Taylor, \& Williams (1996) presented a chemical model for collapsing cloud cores and suggested that the chemical evolutionary age, consistent with the column densities of carbon-chain molecules and $\mathrm{NH}_{3}$, could be deduced for each core in TMC-1. Pratap et al. (1997) carried out intensive mapping observations of several molecular species along the ridge of TMC-1. They found that the observed abundance gradients could be explained by a small variation in the chemical timescale from $1.2 \times 10^{5}$ to $1.8 \times 10^{5} \mathrm{yr}$, by a change in density by a factor of 2 along the ridge, or by a variation in the $\mathrm{C} / \mathrm{O}$ ratio from 0.4 to 0.5 along the ridge. Recently, Markwick, Millar, \& Charnley $(2000)$ presented an interesting explanation to this problem: the density gradients along the ridge were produced following the explosive desorption of photolyzed ices, induced by MHD waves as they propagate inside the cloud.

Several deuterated species are detected toward TMC-1 (Langer et al. 1980; MacLeod, Avery, \& Broten 1981; Schloerb et al. 1981). In 1982, Guélin and his collaborators carried out mapping observations of five isotopic species of $\mathrm{HCO}^{+}$toward several dense molecular clouds including TMC-1. They found that $\mathrm{HCO}^{+}$is distributed in a wide region of the $\mathrm{TMC}-1$ ridge, showing its peak position at about 1 !.5 northwest of the ammonia peak, whereas the peak position of $\mathrm{DCO}^{+}$coincides with the ammonia peak. Later, Butner, Lada, \& Loren (1995) conducted a survey observation of $\mathrm{DCO}^{+}$and $\mathrm{H}^{13} \mathrm{CO}^{+}$in a sample of low-mass cores including the ammonia peak of TMC-1 and determined the $\left[\mathrm{DCO}^{+}\right] /\left[\mathrm{HCO}^{+}\right]$fractionation ratio to be 0.027 for the ammonia peak.

Bell et al. (1988) detected $c-\mathrm{C}_{3} \mathrm{HD}$ toward several molecular clouds. They obtained very high abundance ratios of $c$ $\mathrm{C}_{3} \mathrm{HD}$ to $c-\mathrm{C}_{3} \mathrm{H}_{2}$ in the TMC-1 region: 0.08 at the cyanopolyyne peak, 0.12 in the region of the ammonia peak, and 0.16 in the region of the $\mathrm{HCO}^{+}$peak. This high fractionation of the deuterated species necessitated a reconsideration of the chemical formation routes to $c-\mathrm{C}_{3} \mathrm{H}_{2}$ and $c-\mathrm{C}_{3} \mathrm{HD}$. Howe \& Millar (1993) presented two chemical models that 
give the degree of the deuterium fractionation for $c-\mathrm{C}_{3} \mathrm{H}_{2}$ to be $\sim 0.15$.

In 1994, Howe et al. carried out extensive high-resolution mapping observations of $\mathrm{DC}_{3} \mathrm{~N}$ and $\mathrm{HC}_{3} \mathrm{~N}$ toward several molecular clouds, especially a few positions along the TMC-1 ridge centered at the cyanopolyyne peak. They obtained a high deuterium fractionation of a few to 10 percent, which was explained by linking the deuteration of $\mathrm{HC}_{3} \mathrm{~N}$ to that of $c-\mathrm{C}_{3} \mathrm{H}_{2}$. Recently, we carried out a spectral line survey toward the cyanopolyyne peak of TMC-1 by using the Nobeyama $45 \mathrm{~m}$ radio telescope (Kaifu et al. 2002) and obtained relative abundances for four deuterated species: $\mathrm{DC}_{3} \mathrm{~N}, \mathrm{DC} \mathrm{C}_{5} \mathrm{~N}, \mathrm{HDCS}$, and $c-\mathrm{C}_{3} \mathrm{HD}$. Very recently, Tiné et al. (2000) detected $\mathrm{NH}_{2} \mathrm{D}$ and $\mathrm{N}_{2} \mathrm{D}^{+}$toward the ammonia core and reported abundance ratios of 0.02 and 0.08 with respect to $\mathrm{NH}_{3}$ and $\mathrm{N}_{2} \mathrm{H}^{+}$, respectively. Hirota, Ikeda, \& Yamamoto (2001) carried out mapping observations of $\mathrm{DNC}$ and $\mathrm{HN}^{13} \mathrm{C}$ toward TMC-1 and found $[\mathrm{DNC}] /[\mathrm{HNC}]$ to be 0.028 and 0.042 toward the cyanopolyyne peak and the ammonia peak, respectively. The observed relative abundances of the deuterated species in TMC-1 are summarized in Table 1.

The high chemical fractionation of the deuterated species occurs in the gas phase at low temperatures (Watson 1976, 1977), and the degree of fractionation depends on the time elapsed (Millar, Bennett, \& Herbst 1989; Howe \& Millar 1993). We suggest in this paper that the degree of chemical fractionation of selected molecular species can be used to

TABLE 1

DEUterium Fractionatron IN TMC- $1^{\text {a }}$

\begin{tabular}{|c|c|c|c|}
\hline Molecule & CP Peak & $\mathrm{NH}_{3}$ Peak $^{\mathrm{c}}$ & $\mathrm{HCO}^{+} \mathrm{Peak}^{\mathrm{d}}$ \\
\hline$R\left(\mathrm{HCO}^{+}\right) \ldots \ldots$ & $1.7,{ }^{\mathrm{e}} 1.2^{\mathrm{f}}$ & $2.7^{\mathrm{g}}$ & \\
\hline$R\left(\mathrm{C}_{3} \mathrm{H}_{2}\right) \ldots \ldots \ldots$ & $8,{ }^{\mathrm{h}} 7.5,{ }^{\mathrm{i}} 4.75^{\mathrm{f}}$ & $12,{ }^{\mathrm{h}} 3-6^{\mathrm{j}}$ & $16^{\mathrm{h}}$ \\
\hline$R\left(\mathrm{HC}_{3} \mathrm{~N}\right) \ldots \ldots .$. & $5-6,{ }^{\mathrm{k}} \cdot 3-6,{ }^{\mathrm{l}} 2,{ }^{\mathrm{i}} 1.45^{\mathrm{f}}$ & & \\
\hline$R\left(\mathrm{HC}_{5} \mathrm{~N}\right) \ldots \ldots$. & $1.2,^{\mathrm{m}} 1.6,^{\mathrm{n}} 3^{\mathrm{i}}$ & & \\
\hline$R\left(\mathrm{C}_{2} \mathrm{H}\right) \ldots \ldots \ldots \ldots$ & $4.8(15)^{\mathrm{f}}$ & & \\
\hline$R\left(\mathrm{C}_{4} \mathrm{H}\right) \ldots \ldots \ldots$ & $0.43^{\circ}$ & & \\
\hline$R\left(\mathrm{H}_{2} \mathrm{CO}\right) \ldots \ldots \ldots$ & & $5.9^{f}$ & \\
\hline$R\left(\mathrm{H}_{2} \mathrm{CS}\right) \ldots \ldots \ldots$ & $2^{\mathrm{p}}$ & & \\
\hline$R\left(\mathrm{NH}_{3}\right) \ldots \ldots \ldots$ & $0.085^{f}$ & $2,94.3^{f}$ & \\
\hline$R\left(\mathrm{~N}_{2} \mathrm{H}^{+}\right) \ldots \ldots$ & $0.64(13)^{\mathrm{f}}$ & $8,90.98^{\mathrm{i}}$ & \\
\hline$R(\mathrm{HCN}) \ldots \ldots \ldots$ & $1.10(46)^{f}$ & & \\
\hline$R(\mathrm{HNC}) \ldots \ldots \ldots$ & $2.8,{ }^{r} 1.6^{\mathrm{f}}$ & $4.2^{r}$ & \\
\hline$R\left(\mathrm{CH}_{3} \mathrm{CCH}\right) \ldots$ & $5.1-6.1^{\mathrm{s}}$ & & \\
\hline$R\left(\mathrm{CH}_{3} \mathrm{OH}\right) \ldots$. & $2.65^{\mathrm{f}}$ & & \\
\hline
\end{tabular}

a $R(\mathrm{XH})=[N(\mathrm{XD})] /[N(\mathrm{XH})] \times 100$.

b Cyanopolyyne peak, $\alpha=4^{\mathrm{h}} 38^{\mathrm{m}} 38^{\mathrm{s}} \cdot 6, \delta=25^{\circ} 35^{\prime} 45^{\prime \prime}(1950.0)$.

c $a=4^{\mathrm{h}} 38^{\mathrm{m}} 19 \mathrm{~s} 0, \delta=25^{\circ} 42^{\prime} 30^{\prime \prime}(1950.0)$

${ }^{d} \alpha=4^{\mathrm{l}} 38^{\mathrm{m}} 12^{\mathrm{s}} \cdot 0, \delta=25^{\circ} 44^{\prime} 00^{\prime \prime}(1950.0)$.

e Caselli et al. 1998

1 Turner 2001. Values in parentheses denote the errors quoted and apply to the last digits of the relative abundance ratios.

g Butner et al. 1995

h Bell et al. 1988.

i Kaifu et al. 2001.

j Gerin et al. 1987.

k Langer et al. 1980.

${ }^{1}$ Howe et al. 1994

m Macleod et al. 1981

"Schloerbet al. 1981.

- Turner 1989.

p Minowa et al. 1997.

q Tiné et al. 2000

${ }^{r}$ Hirota et al. 2001.

s Gerin et al. 1992, referred to $\mathrm{CH}_{2} \mathrm{DCCH}$ estimate the chemical evolutionary age of dark cloud cores. In a similar but more comprehensive vein, Roberts $\&$ Millar (2000a) published a paper on the modeling of deuterium chemistry. They presented new models for investigating deuterium fractionation in molecular clouds with a wide range of physical parameters, including density, temperature, elemental abundances, and the freeze-out of molecules on to dust grains, and they showed how fractionation can be used to probe the history of interstellar matter.

\section{CHEMICAL EVOLUTIONARY AGE OF CORES IN TMC-1}

Only three deuterated species, $\mathrm{DCO}^{+}, c-\mathrm{C}_{3} \mathrm{HD}$, and DNC, have been studied so far at both the cyanopolyyne peak and the ammonia peak of the TMC-1 ridge, where physical conditions are relatively well known (Table 1). $\mathrm{HCO}^{+}$is one of the most fundamental molecular ions in interstellar gaseous chemistry, and $\mathrm{DCO}^{+}$is a primary species in interstellar deuterium fractionation, to which the deuterium atom is transferred from highly fractionated $\mathrm{H}_{2} \mathrm{D}^{+}$at low temperatures $(\sim 10 \mathrm{~K})$. Both $\mathrm{HCO}^{+}$and $\mathrm{DCO}^{+}$have been detected and studied in a wide variety of molecular clouds, from dark clouds to massive star-forming regions.

As noted above, $c-\mathrm{C}_{3} \mathrm{HD}$ shows a very high relative abundance as well as a large change along the TMC-1 ridge. However, Turner (2001) recently reported that his analyses of $\mathrm{C}_{3} \mathrm{H}_{2}$ and $\mathrm{C}_{3} \mathrm{HD}$ disagreed with the earlier analyses, and much lower fractionation values were derived for TMC-1 cores. The new analyses are consistent with a new prediction that the key reaction to produce the $\mathrm{C}_{3} \mathrm{HD}$ species,

$$
\mathrm{C}_{3} \mathrm{H}_{3}^{+}+\mathrm{HD} \rightarrow \mathrm{C}_{3} \mathrm{H}_{2} \mathrm{D}^{+}+\mathrm{H}_{2},
$$

has a potential barrier of about $4 \mathrm{eV}$ and does not contribute at all to deuterium fractionation at temperatures less than $10 \mathrm{~K}$ (Talbi \& Herbst 2001).

Although the relative abundance between DNC and HNC was observationally determined with relatively good accuracy, the production and fractionation of these species have an ambiguity, and the relative abundance is not suitable for quantitative analysis, because the branching ratio in dissociative recombination of $\mathrm{HCND}^{+}$is not known. Furthermore, several chemical model calculations so far presented show a large hump of the DNC abundance around $10^{5}$ to $10^{6}$ ages (for an example, Howe \& Millar 1993). This means that a given relative abundance of DNC corresponds to two separate years for the limited period.

The relative abundance of $\mathrm{DCO}^{+}$at the cyanopolyyne peak was reported to be $1.7 \% \pm 0.3 \%$ by Caselli et al. (1998) using the data of $\mathrm{DCO}^{+}$and $\mathrm{H}^{13} \mathrm{CO}^{+}$by Guélin, Langer, \& Wilson (1982), and that at the ammonia peak to be $2.7 \% \pm 0.5 \%$ using the data by Butner et al. (1995). Caselli et al. (1998) analyzed the abundance ratios $R_{\mathrm{D}}=\left[\mathrm{DCO}^{+}\right] /$ $\left[\mathrm{HCO}^{+}\right]$and $R_{\mathrm{H}}=\left[\mathrm{HCO}^{+}\right] /[\mathrm{CO}]$ of several dark cloud cores so as to determine the electron fraction, $\chi(e)$, the cosmic-ray ionization rate, $\zeta$, and the $\mathrm{C} / \mathrm{O}$ depletion degree, $f_{\mathrm{D}}$. The depletion factor, $f_{\mathrm{D}}$, is defined such that a fraction $1 / f_{\mathrm{D}}$ of the $\mathrm{O}$ and $\mathrm{C}$ in the standard "low metal" abundances remains in the gas phase. Their analysis is based on a simple analytical chemical model of deuterium fractionation and the steady state abundances derived from a detailed pseudo-time-dependent chemical model. They derived 
physical conditions at both the cyanopolyyne peak and the ammonia peak, but the steady state physical condition at the cyanopolyyne peak is inappropriate, as they pointed out, because their steady state analysis cannot reproduce the abundances of complex molecules observed toward TMC-1(CP). Pratap et al. (1997) reported that the kinetic temperature along the TMC-1 ridge is derived to be $10 \mathrm{~K}$ from $\mathrm{CH}_{3} \mathrm{CCH}$ and $\mathrm{NH}_{3}$ lines, which is almost consistent with the temperature derived from ${ }^{12} \mathrm{CO}$ and ${ }^{13} \mathrm{CO}$ lines. They also derived the molecular hydrogen density, $n\left(\mathrm{H}_{2}\right)$, to be $8.3 \times 10^{4}$ and $6.2 \times 10^{4} \mathrm{~cm}^{-3}$ at the $\mathrm{CP}$ and $\mathrm{NH}_{3}$ peaks, respectively, from the analysis of $\mathrm{HC}_{3} \mathrm{~N}$ lines.

The degree of deuterium fractionation depends on the physical conditions of the cloud core where molecules evolve. It is essential to use plausible physical conditions for a quantitative analysis of deuterium fractionation. As noted above, Caselli et al. (1998) carried out an extensive and intensive analysis of physical conditions for many dark clouds, varying the ionization rate. We think the ionization rate does not change far from the standard value, $1.3 \times 10^{-17} \mathrm{~s}^{-1}$, in such dark cloud cores. No ultraviolet radiation sources along the TMC-1 ridge are known, and the cosmic-ray ionization rate can be safely assumed to be the standard value. If the molecular hydrogen densities are assumed to be those obtained by Pratap et al. (1997) for the $\mathrm{CP}$ and $\mathrm{NH}_{3}$ cores, the simple analytical chemical model used by Caselli et al. (1998), that is, an analysis using equations (3) and (4) of their paper, gives $f_{\mathrm{D}}$ to be about 1 based on the observed $R_{\mathrm{D}}$ and $R_{\mathrm{H}}$ values toward both peaks. Therefore, it is reasonable to choose physical conditions for both cores to be $T_{\mathrm{K}}=10 \mathrm{~K}, n\left(\mathrm{H}_{2}\right)=$ $7 \times 10^{4} \mathrm{~cm}^{-3}, \zeta=1.3 \times 10^{-17} \mathrm{~s}^{-1}$, and $f_{\mathrm{D}}=1$, physically static conditions, which are used to calculate the relative abundance of $\left[\mathrm{DCO}^{+}\right] /\left[\mathrm{HCO}^{+}\right]$by employing the new standard model network of gas-phase chemical reactions (Terzieva \& Herbst 1998). The network has been extended to include monodeuterated species of hydrogen-bearing molecules (Millar et al. 1989; Aikawa \& Herbst 1999). For normal exothermic reactions and dissociative recombination reactions, the total rate coefficient is assumed to be unchanged for deuterated species and statistical branching ratios are also assumed. $\mathrm{HCO}^{+}\left(\mathrm{DCO}^{+}\right)$is mainly produced by the reaction of $\mathrm{CO}$ with $\mathrm{H}_{3}^{+}\left(\mathrm{H}_{2} \mathrm{D}^{+}\right)$and destroyed by the dissociative recombination reaction with electrons (Dalgarno \& Lepp 1984). In total, 639 molecular species and 10,830 chemical reactions are included in the network. The model is pseudo-time-dependent, and we assumed an initial state where all elements except hydrogen are atomic with "low metal" abundances of $\chi(\mathrm{O})=1.8 \times 10^{-4}$, $\chi(\mathrm{C})=7.3 \times 10^{-5}, \chi\left(\mathrm{S}^{+}\right)=8 \times 10^{-8}, \quad \chi\left(\mathrm{Si}^{+}\right)=8 \times 10^{-9}$, $\chi\left(\mathrm{Mg}^{+}\right)=7 \times 10^{-9}, \quad \chi\left(\mathrm{Fe}^{+}\right)=3 \times 10^{-9}$, and $\chi\left(\mathrm{Na}^{+}\right)=$ $2 \times 10^{-9}$. All deuterium was assumed to be present as HD in the initial conditions and its fractional abundance to be $3.0 \times 10^{-5}$ (Millar et al. 2000). The calculated relative abundance ratio $R\left(\mathrm{HCO}^{+}\right)=100 \times R_{\mathrm{D}}$ is shown by the curve (a) in Figure 1 as a function of time, where the observed relative abundances with error bars are also indicated at the right-hand side of the figure.

As Figure 1 shows, there is a difference of about $0.5 \%$ in the relative abundance between the observed value toward the ammonia peak and the predicted value at steady state, though the observed values show relatively large errors. If we assume that the observed value toward the ammonia peak corresponds to the steady state value, then, if the $0.5 \%$

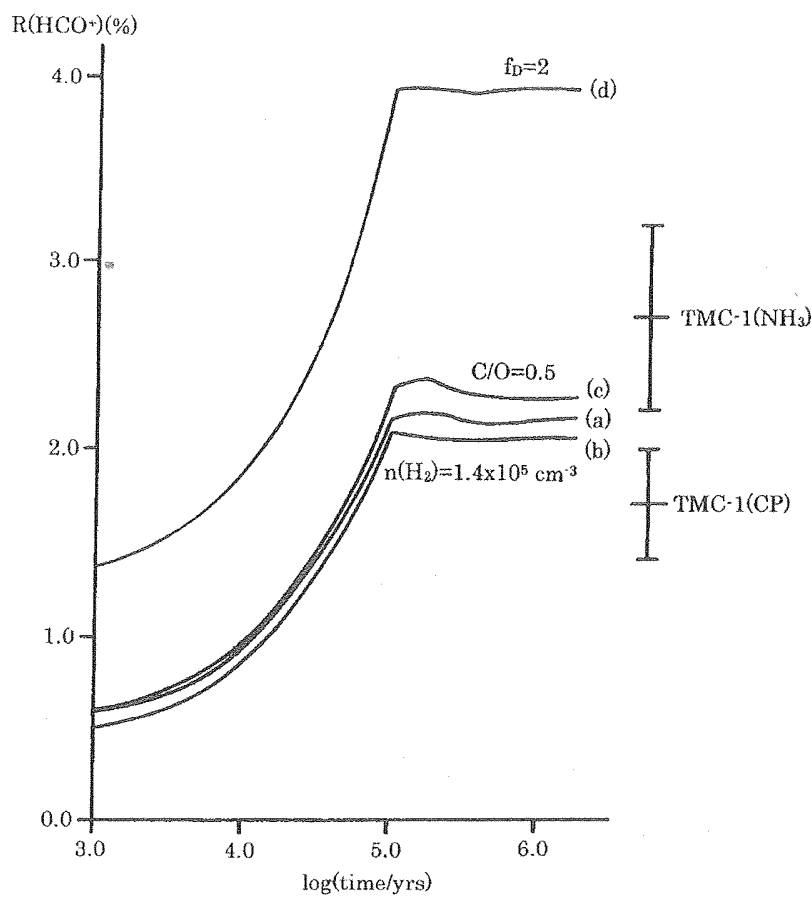

FIG. 1. - The calculated relative abundance ratio between $\mathrm{DCO}^{+}$and $\mathrm{HCO}^{+}, R\left(\mathrm{HCO}^{+}\right)\left[=100 \times R_{\mathrm{D}}\right.$ (Caselli et al. 1998)], using the new standard model network with $\zeta=1.3 \times 10^{-17} \mathrm{~s}^{-1}$, and "low metal" abundances of $\quad \chi(\mathrm{O})=1.8 \times 10^{-4}, \quad \chi(\mathrm{C})=7.3 \times 10^{-5}, \quad \chi\left(\mathrm{S}^{+}\right)=8 \times 10^{-8}$, $\chi\left(\mathrm{Si}^{+}\right)=8 \times 10^{-9}, \quad \chi\left(\mathrm{Mg}^{+}\right)=7 \times 10^{-9}, \quad \chi\left(\mathrm{Fe}^{+}\right)=3 \times 10^{-9}, \quad$ and $\chi\left(\mathrm{Na}^{+}\right)=2 \times 10^{-9}$ at $10 \mathrm{~K}$. Curve (a): $n\left(\mathrm{H}_{2}\right)=7 \times 10^{4} \mathrm{~cm}^{-3}, f_{\mathrm{D}}=1$, and $\mathrm{C} / \mathrm{O}=0.4$; curve (b) $n\left(\mathrm{H}_{2}\right)=1.4 \times 10^{5} \mathrm{~cm}^{-3}, f_{\mathrm{D}}=1$, and $\mathrm{C} / \mathrm{O}=0.4$; curve (c) $\mathrm{C} / \mathrm{O}=0.5, n\left(\mathrm{H}_{2}\right)=7 \times 10^{4} \mathrm{~cm}^{-3}$ and $f_{\mathrm{D}}=1$, and curve (d) $f_{\mathrm{D}}=2, n\left(\mathrm{H}_{2}\right)=7 \times 10^{4} \mathrm{~cm}^{-3}$, and $\mathrm{C} / \mathrm{O}=0.4$. The observed values at the cyanopolyyne peak and the ammonia peak are indicated with error bars at the right thand side.

difference is time-independent, that of the cyanopolyyne peak is deduced to correspond to the relative abundance at $1.6 \times 10^{4} \mathrm{yr}$. Since steady state pertains at $\geq 1 \times 10^{5} \mathrm{yr}$, it may be concluded that the difference between the evolutionary ages between the ammonia peak and the cyanopolyyne peak is probably more than $10^{5} \mathrm{yr}$.

\section{DISCUSSION}

Suzuki et al. (1992) showed that carbon-chain molecules including CCS are abundant in the early stages of chemical evolution, whereas $\mathrm{NH}_{3}$ is abundant in the later stages, on the basis of pseudo-time-dependent chemical reaction network simulations. The conclusion suggests that the cyanopolyyne peak of the TMC-1 ridge is in an earlier evolutionary stage than the ammonia peak. Although it is qualitative, this suggestion was reinforced by Hirahara et al. (1992), who carried out high-resolution mapping observations of several molecules along the TMC-1 ridge and showed a gradual change in the ratio between the column densities of $\mathrm{NH}_{3}$ and carbon-chain molecules along the ridge. The sense of the present study, that the ammonia peak is older than the cyanopolyyne peak, is in qualitative agreement with the standard gas-phase models which show that CCS peaks at early times and ammonia peaks at steady state. 
From their intensive mapping observations along the TMC-1 ridge, Pratap et al. (1997) concluded that the observed abundance gradients along the ridge can be explained by a small variation in the chemical timescale from $1.2 \times 10^{5}$ to $1.8 \times 10^{5} \mathrm{yr}$. Their conclusion is based on the relative abundance between $\mathrm{CS}$ and SO along the ridge, which is suggested to be a very sensitive indicator of the evolutionary state of the cloud (Bergin et al. 1997), according to time-dependent chemical model calculations (Bergin et al. 1995; Bergin, Snell, \& Goldsmith 1996). They also suggested alternatively that the observed gradients can be produced by varying the density by a factor of 2 or the $\mathrm{C} / \mathrm{O}$ ratio from 0.4 to 0.5 along the ridge.

We investigated the dependence of the abundance ratio, $R_{\mathrm{D}}$, on physical parameters, using the new standard model. A change in the density by a factor of 2 gives only a change of less than $0.1 \%$ in the abundance ratio, as indicated in the curve (b) of Figure 1. A change of the $\mathrm{C} / \mathrm{O}$ ratio from 0.4 to 0.5 produces only a similar small change of less than $0.2 \%$, as illustrated in the curve (c) of Figure 1. Pratap et al. (1997) suggest that the high $\mathrm{C} / \mathrm{O}$ ratio reproduces high column densities of carbon-bearing species at the $\mathrm{CP}$ core, whereas the low $\mathrm{C} / \mathrm{O}$ value prefers oxygen-bearing species at the $\mathrm{NH}_{3}$ core. However, this figure shows that the change in $R_{\mathrm{D}}$ as $\mathrm{C} / \mathrm{O}$ varies from 0.4 to 0.5 is contrary to the direction of change of observed values at the $\mathrm{CP}$ to the $\mathrm{NH}_{3}$ cores. Similarly, a change in the ionization rate or the metal abundance by a factor of 2 , which is exemplified in the analysis of Case1 li et al. (1998) for the two peaks of TMC-1, also gives only a small change of less than $0.2 \%$ in the abundance ratio, $R_{\mathrm{D}}$, from $10^{4} \mathrm{yr}$ to the steady state. An increase of the depletion factor of carbon and oxygen raises the relative abundance to a higher value. For example, the relative abundance of $\mathrm{DCO}^{+}$increases by $1.0 \%-1.5 \%$ when $f_{\mathrm{D}}$ is increased from 1 to 2 , as shown in the curve (d) of Figure 1 . The observed $R_{\mathrm{D}}$ variation between the peaks can therefore be explained by introducing an $f_{\mathrm{D}}$ value between 1 and 2 . However, $f_{\mathrm{D}}$ may also change during the physical evolution of the cloud core; the more cloud evolves, the higher $f_{\mathrm{D}}$. This means that $f_{\mathrm{D}}$ is an indicator of the evolutionary state of the core, as pointed out by Roberts \& Millar (2000a, 2000b). A temporal increase in $f_{\mathrm{D}}$ thus raises calculated $R_{\mathrm{D}}$ values at late times and moves them closer to the observed values at the $\mathrm{NH}_{3}$ peak.

Therefore, we conclude that the molecular abundance gradients and the variation of the degree of deuterium fractionation along the TMC-1 ridge can be explained by a chemical evolutionary age difference of more than $10^{5} \mathrm{yr}$ or a small increase in the depletion factor of carbon and oxygen, which is itself likely an effect of evolution. Based on the recent mapping observations of $\mathrm{DNC}$ and $\mathrm{H}^{13} \mathrm{NC}$ toward nearby dark cloud cores including the TMC-1 ridge, Hirota et al. (2001) also suggested qualitatively that the systematic variation of the deuterium fractionation could be interpreted in terms of the chemical evolution of dark cloud cores.

It may be interesting to compare the present evolutionary age difference of more than $10^{5} \mathrm{yr}$ between the ammonia peak and the cyanopolyyne peak with the results obtained from other models. Howe, Taylor et al. (1996) inferred the age difference between the cores to be of the order of $3 \times 10^{5}$ yr by applying the observed column densities of CCS and its related species to their sequential core collapse model.
Recently, Markwick et al. (2000) proposed another model to explain the observed gradients along the ridge. The protostellar object IRAS $04381+2540$ near the ammonia peak produces MHD waves. These Alfvén waves trigger the spontaneous explosion of UV-photolyzed grain mantles by heating the dust grains and their ice mantles through grain-grain collisions. The removal of icy grain mantles restarts the chemical processes in the cores, first in the ammonia core and later in the CP core. The propagation time difference from the IRAS source to the cloud cores is $1.5 \times 10^{5}$ yr assuming the speed of MHD waves to be $2 \mathrm{~km} \mathrm{~s}^{-1}$. This dynamical chemical model produces the gradients in molecular abundances along the TMC-1 ridge. It is interesting to see how deuterium fractionation at both peaks varies with this model, an analysis which has not yet been undertaken. Ambipolar diffusion tends to suppress the contribution of $\mathrm{H}_{2} \mathrm{D}^{+}$to gas phase deuterium fractionation (Markwick et al. 2000), although there is an initially high deuterium concentration just after the grain mantle explosion that declines on a timescale of $10^{5} \mathrm{yr}$ (Charnley, Tielens, \& Millar 1992).

When this paper was in the reviewing process, we learned that Turner (2001) carried out observations of several deuterated species in the dark clouds including TMC-1 and determined precise relative abundances of the deuterated species from an analysis using reliable radiative transport models with microturbulent cloud structure. He reported $R\left(\mathrm{HCO}^{+}\right)$only at the cyanopolyyne peak to be $1.2 \%$, which could be compared with $1.7 \% \pm 0.3 \%$ (Caselli et al. 1998). He also studied the chemistry of deuterated species using the modified New Standard Model with several different physical conditions but could not obtain a good match at steady state between the observed and calculated $R\left(\mathrm{HCO}^{+}\right)$ for TMC-1CP.

In conclusion, it is shown that the degree of deuterium fractionation of $\mathrm{HCO}^{+}$is a plausible candidate to estimate the chemical evolutionary age of dark cloud cores, even though the chemical model used in the present study suffers from some ambiguity in the physical conditions applied, and the errors in the observed relative abundances of $\mathrm{DCO}^{+}$ are relatively large. Our analysis is based on the fact that the production and destruction of $\mathrm{HCO}^{+}$and $\mathrm{DCO}^{+}$are well known and relatively simple, and somewhat free from the details of chemical reactions not directly related to deuterium fractionation. The fractionation of $\mathrm{DCO}^{+}$thus provides a better constraint on the evolutionary state than other molecular abundances and other deuterated species. If several deuterated species as well as their parent species are observed toward dark cloud cores where physical conditions are well known, we can choose chemical species especially sensitive in their degree of deuterium fractionation to evolutionary age. Furthermore, we can check the reliability of the estimated evolutionary age by using multiple molecular species. Here it must be emphasized that a very reliable chemical model as well as plausible physical conditions are essential in the evolutionary age determination of dark cloud cores.

The present study was supported by Grants-in-Aid from the Ministry of Education and Science (no. 12440161) and from Japan Space Forum ("Ground-based Research for Space Utilization" programs). 


\section{REFERENCES}

Aikawa, Y., \& Herbst, E. 1999, ApJ, 526, 314

Bell, M. B., Avery, L. W., Matthews, H. E., Feldman, P. A., Watson, J. K.

G., Madden, S. C., \& Irvine, W. M. 1988, ApJ, 326, 924

Bergin, E. A., Goldsnith, P. F., Snell, R. L., \& Langer, W. D. 1997, ApJ, 482,285

Bergin, E. A., Langer, W. D., \& Goldsmith, P. F. 1995, ApJ, 441, 222

Bergin, E. A., Snell, R. L., \& Goldsmith, P. F. 1996, ApJ, 460, 343

Butner, H. M., Lada, E. A., \& Loren, R. B. 1995, ApJ, 448, 207

Caselli, P., Walmsley, C. M., Terzieva, R., \& Herbst, E. 1998, ApJ, 499, 234

Charnley, S. B., Tielens, A. G. G. M., \& Millar T. J. 1992, ApJ, 399, L71

Gerin, M., Combes, F., Wlodarczak, G., Encrenaz, P., \& Laurent, C. 1992 ,$$
\text { A\&A, 253, L29 }
$$

Gerin, M., Wootten, H. A., Combes, F., Boulanger, F. Peters II, W. L.,

Kuiper, T. B. H., Encrenaz, P. J., \& Bogey, M. 1987, A\&A, 173, L1

Guélin, M., Langer, W. D., \& Wilson, R. W. 1982, A\&A, 107, 107

Dalgarno, A., \& Lepp, S. 1984, ApJ, 287, L47

Hanawa, T., Yamamoto, S., \& Hirahara, Y. 1994, ApJ, 420, 318

Hasegawa, T. I., \& Herbst, E. 1993, MNRAS, 261, 83

Herbst, E., Lee, H.-H., Howe, D. A., \& Millar, T, J. 1994, MNRAS, 268 , 335

Herbst, E \& Leung C. M. 1989, ApJS, 69, 271 1990, A\&A, 233, 177

Hirahara, Y., et al. 1992, ApJ, 394, 539

Hirota, T., Ikeda, M., \& Yamamoto, S. 2001, ApJ, 547, 814

Howe, D. A., \& Millar, T. J. 1993, MNRAS, 262, 868

Howe, D. A., Millar, T. J., Schilke, P., \& Walmsley, C. M. 1994, MNRAS,

267,59

Howe, D. A., Taylor, S. D., \& Williams, D. A. 1996, MNRAS, 279, 143
Kaifu, N., et al. 2002, PASJ, submitted

Langer, W. D., Schloerb, F. P., Snell, R. L., \& Young, J. S. 1980, ApJ, 239, L125

Lee, H.-H., Bettens, R P. A \& Herbst, E 1996, A\&AS, 119, L111

MacLeod, J. M. Avery, L. W. \& Broten, N.W 1981, ApJ, 251, L33

Markwick, A. J., Millar, T. J., \& Charnley, S. B. 2000, ApJ, 535, 256

Millar, T. J., Bennett, A., \& Herbst E. 1989 , ApJ, 340,906

Millar, T. J., Roberts, H., Markwick, A. J., \& Charnley, S. B. 2000, Phil. Trans. R. Soc. Lond. A, 358, 2535

Minowa, H., Satake, M., Hirota, T., Yamamoto, S., Ohishi, M., \& Kaifu, N. 1997, ApJ, 491, L63

Pratap, P.. Dickens, J. E., Snell, R. L. Miralles, M. P., Bergin, E. A., Irvine,

W. M., \& Schloerb, F.P. 1997, ApJ, 486, 862

Roberts, H., \& Millar, T. J. 2000a, A\&A, 361, 388 2000 b, A\&A, 364, 780

Schloerb, F. P., Snell, R. L., Langer, W. D., \& Young, J. S. 1981, ApJ, 251, L37

Suzuki, H., Yamamoto, S., Ohishi, M., Kaifu, N., Ishikawa, S., Hirahara, Y., \& Takano, S. 1992, ApJ, 392, 551

Talbi, D. \& Herbst, E. 2001 A\&A, 376,663

Terzieva, R., \& Herbst, E. 1998, ApJ, 501, 207

Tiné, S., Roueff, E., Falgarone, E., Gerin, M., \& Pineau des Foréts, G. 2000, A\&A, 356, 1039

Turner, B. E. 1989 , ApJ, 347, L39

.2001, ApJS, 136,579

Watson, W. D. 1976, Rev. Mod. Phys., 48, 513

1977, in CNO Processes in Astrophysics, ed. J. Audonze (Dordrecht: Reidel), 105 